\title{
CALIBRATING THE TEM
} John P. McCaffrey, National Research Council of Canada

An accurate calibration of magnification, diffraction patterns and the relative rotation between the two is critical in quantitative transmission electron microscopy (TEM). The manufacturers of TEM's supply magnification values for each image magnification step and each value of camera length for their instruments, but these values have been observed to be in error by up to $10 \%$. These errors can occur due to variations in lenses in individual microscopes as well as through power supply variations and aging electronic components. Before attempting quantitative TEM analysis, a series of calibrations of the individual TEM should be undertaken. Historically, four or five separate calibration samples were required to properly calibrate a transmission electron microscope (see Edington, (1976) for an excellent review). More comprehensive calibration samples are now being manufactured using the techniques of the semiconductor industry that allow the three major TEM calibrations to be performed with a single sample (McCaffrey, 1995).

For the magnification calibration of a TEM, the general procedure is to take micrographs of standard samples with known spacings at all magnification values accessible by that standard. Most standards are only suitable for a specific magnification range. The images of these known spacings are then measured on the film negative or CCD display, and an accurate value of the magnification at that particular magnification step is calculated. The result is a table of values:

Nominal (TEM display) Magnification:

$\begin{array}{rr}3,900 X & 3,600 X \\ 13,600 X & 14,800 X \\ 52,100 X & 47,600 X \\ 122,000 X & 115,000 X\end{array}$

Until recently, a single sample could not cover all TEM magnification ranges, so a wide variety of techniques and samples have been used to provide accurate values for partial ranges of magnification steps. These include diffraction grating replicas (for example, Oster and Skillman, 1962), direct lattice images (Dowell, 1964), latex spheres (Backus and Williams, 1948), metal mesh (Skinner, 1969), and moiré patterns (Dowell, 1964). Lattice images of crystals (usually gold islands) provided the most accurate calibration at the highest magnifications, but at magnifications under about $400,000 \mathrm{X}_{1}$ they became increasingly difficult to measure. Ruled diffraction grating replicas typically provided spacings of approximately $400 \mathrm{~nm}$ - a useful calibration for magnifications in the $1,000 \mathrm{X}$ to $50,000 \mathrm{X}$ range. The intermediate range of $50,000 X$ to $400,000 X$ was usually calibrated with samples such as catalase (Wrigley, 1968), but this particular type of reference material varies significantly in the reference spacing, and cannot be used as a reliable absolute measurement. While most of these calibration samples performed well in their respective magnification ranges, there was generally some difficulty experienced in "stitching together" an accurate calibration over all ranges.

New types of calibration samples can now be made with the technology of the semiconductor industry. One example consists of a cross-section of single-crystal silicon, with a series of calibration marks incorporated into the crystal. When viewed in cross-section in a TEM, it appears as a series of dark silicon-germanium (SiGe) alloy bands alternating with lighter pure silicon bands, all grown on a single-crystal silicon substrate (Fig. 1). The band widths and spacings are chosen to accommodate all of the high, medium and low TEM magnification ranges. One major advantage of this type of calibration sample is that the sample can be self-calibrated to accuracies well beyond the resolution of a TEM. On this sample, all individual bands can be accurately measured with reference to the $\{111\}$ lattice spacing of $\mathrm{Si}_{1}(0.3135428 \mathrm{~nm})$ which is measured on the single-crystal silicon sample itself. This internal consistency allows high confidence levels

in measurement accuracy.

In performing a magnification calibration, special care must be taken in several areas. The calibration sample and all samples on which measurements are being made must be imaged at exactly the eucentric height of the microscope to avoid errors. Hysteresis in the electromagnetic lenses can also cause erroneous measurements. To avoid hysteresis, a useful procedure is to turn the magnification of the microscope to its highest value, then lower the magnification to the range of interest, both when recording the known spacings on the calibration sample, and when making a measurement.

To obtain values for the TEM camera constants that allow indexing electron diffraction patterns, it is necessary to calibrate diffraction patterns at the various camera lengths. Once the camera constant is known for a particular camera length, measurements can be made of diffraction spot or ring spacings, and the corresponding crystal lattice spacing calculated. To perform this calibration, a representative area of the sample is positioned in the TEM, avoiding ambiguous regions, grids, etc. Using the same cautions employed in performing a magnification calibration, diffraction patterns at all camera lengths should be recorded on film or captured with a CCD camera. By measuring either across a polycrystalline diffraction ring and dividing by two, or measuring a series of single crystal spots and dividing by the number of spacings, the value for $R$ in the camera length equation can be determined, and a table of camera constant values can be compiled:

$$
\begin{array}{ll}
\lambda L=d R & \\
\text { where: } & \lambda=\text { wavelength of accelerating voltage }(\mathrm{nm}) \\
& L=\text { Camera length }(\mathrm{mm}) \\
& d=\text { crystal lattice spacing of material }(\mathrm{nm}) \\
& R=\text { diffraction ring radius on negative }(\mathrm{mm})
\end{array}
$$

Knowing the values for " $\mathrm{d}$ " for the calibration sample, and having measured " $R$ ", the camera constant $(\lambda L)$ value can be calculated for each camera length and compiled as a chart

\section{Camera length (TEM display):}

$500 \mathrm{~mm}$

$700 \mathrm{~mm}$

$950 \mathrm{~mm}$

etc

To use this chart of camera constant values to determine unknown crystal lattice plane spacings, diffraction patterns of the unknown material should be recorded at a value of camera length for which the camera constant has been calculated. The unknown lattice spacing is calculated by measuring the diffraction spot spacing or diffraction ring radius " $R$ " on the negative or digital image as above. By then dividing the appropriate camera constant value $\lambda L$ by the measured "R", one arrives at the crystal lattice spacing " $d$ ".

Diffraction pattern calibrations usually require an amorphous compound or pure element sample (thallous chloride, $\mathrm{Al}$, or $\mathrm{Au}$ ) with known, unambiguous spacings. The major source of error in this procedure is the accuracy of the measurement determining these diffraction ring radii (" $R$ " values for different reflections). This value can be measured much more accurately by using the same single crystal silicon sample as was used for the magnification calibration. By choosing a small selected area diffraction aperture, diffraction patterns with sharp, single crystal diffraction spots can be taken at each camera length of interest. By measuring the length of a series of spots and dividing by the total number of spacings (for example, by measuring on the diffraction pattern from the ( bar- 3 bar- 33 ) spot to the ( 33 bar- 3 ) spot and dividing by 6 (see figure 2) a more accurate value of the diffraction ring radius $R$ can be determined.

Finally, the calibration of the rotation between the diffraction pattern and the image is essential for transferring crystallographic information from the diffraction pattern to the image. This is useful for applications such as indexing dislocations or identifying the orientation of crystallites. In TEM's where compensation circuitry is not included, magnetic lenses produce a rotation between an image and the object itself. This rotation depends on lens excitation, so the image rotates on the viewing screen as the magnification is changed. Diffraction 
patterns at a specific camera length, however, are always produced with the same lens excitation, and remain stationary. The calibration of the relative rotation between images and diffraction patterns results in a chart of rotation values for camera length versus magnification, for all camera lengths and magnifications. To produce this chart requires that double exposures of a sample and its diffraction pattern be taken for all rotation values required. By measuring a specific angle consistently in one direction ( $\mathrm{CW}$ or $\mathrm{CCW}$ ), one can compile a chart of rotation values for each magnification and camera length:

\section{Degrees clockwise from diffraction pattern to image direction}

Mag. $\Rightarrow$
Camera
Length

$\begin{array}{cccccc}500 \mathrm{~mm} & 165^{\circ} & 161.5^{\circ} & 256^{\circ} & 246.5^{\circ} & \ldots \\ 700 \mathrm{~mm} & 38^{\circ} & 34.5^{\circ} & 129^{\circ} & 119.5^{\circ} & \ldots \\ 950 \mathrm{~mm} & 40.5^{\circ} & 37^{\circ} & 131.5^{\circ} & 12^{\circ} & \ldots \\ \text { etc. } & \ldots & \ldots & \ldots & \ldots & \ldots\end{array}$

A useful A protocol for these measurements is "degrees clockwise from the direction on the diffraction pattern to the corresponding direction on the image"; (i.e., from a vector pointing from the $(000)$ to the $(200)$ diffraction spot on the diffraction pattern, rotate $26^{\circ}$ clockwise about the $(000)$ spot to find the vector pointing in the [200] direction on the image. From the (220) spot, rotate $26^{\circ}$ clockwise to the (220) image direction; etc. (see Figure 3).

An $\alpha-\mathrm{MoO}_{3}$ sample has traditionally been used for the image/diffraction pattern rotation calibration because of its distinctive shape. The cross-sectional single-crystal silicon sample mentioned above can also be used, since the original sample surface direction can be used as the reference for direction on the

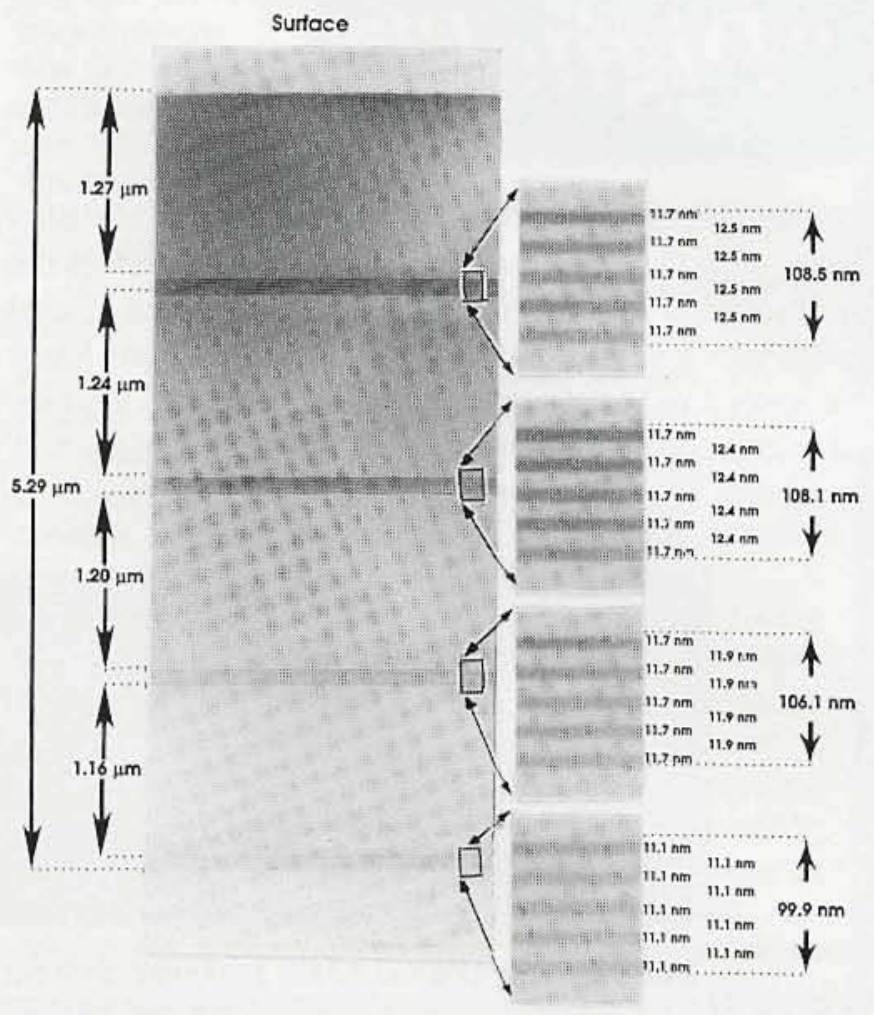

Figure 1: Semiconductor TEM calibration sample, with calibration markings allowing for calibrations at all magnification levels. All markings are directly referenced to the $\{111\}$ lattice spacing of silicon, which can be imaged on the sample itself. (MAG*|*CAL calibration sample courtesy of South Bay Technology Inc., San Clemente, CA) sample. In both of these materials, simple techniques can be used to resolve the $180^{\circ}$ sample ambiguity, i.e., the two-fold crystal symmetry of the viewing zone.

The stability and durability of TEM's is such that once a complete calibration is done, frequent recalibration is not necessary. However, any changes to the high tension supply (high tension cables, power supplies, EPROMS, etc.), call for a recalibration. As a rule, an annual verification or recalibration is prudent.

Backus, R.C. and Williams, R.C. (1948) "Small spherical particles of exceptionally uniform size". J. Appl. Phys., 20:224-225.

Dowell, W.C.T. (1964) "Die bestimmung der vergoBerung des elektronenmikroskops mittels elektroneninterferenz". Optik, 21: 26-40.

Edington, J.W. (1976) Practical Electron Microscopy in Materials Science, (Van Nostrand Reinhold, New York), 21-30.

Mccaffrey, J.P. and Baribeau, J-M. (1995) "A transmission electron microscope (TEM) calibration standard sample for all magnification, camera constant and imagediffraction pattern rotation calibrations", Microscopy Research and Technique, 32 no.5, pp. 449-454.

Oster, Jr. C.F. and Skillman, D.C., (1962) Proc, 5th Intern. Congr. for Electron Microscopy, vol.1, Academic Press, New York, (1962) EE-3-5.

Skinner, L.M. (1969) "An accurate method for measuring the magnification of an electron microscope". J. Phys. E., 2: 206-208.

South Bay Technology Inc., 1120 Via Callejon, San Ciemente, CA 92673, tel: (714) 492-2600, e-mail: henriks@southbaytech.com, http://www. southbaytech.com

Wrigley, N.G. (1968), The lattice spacing of crystalline catalase as an internal standard of length in electron microscopy, J. Ultrastructure Res., 24: 454-464.

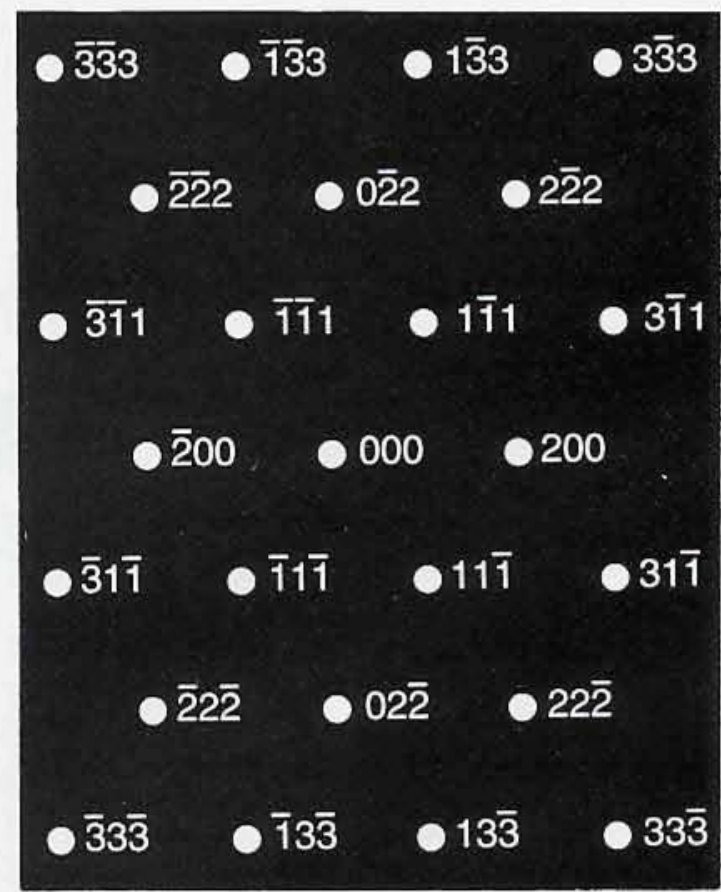

Figure 2: Diagram of an indexed cubic electron diffraction pattern viewed down the $\langle 011\rangle$ zone axis.

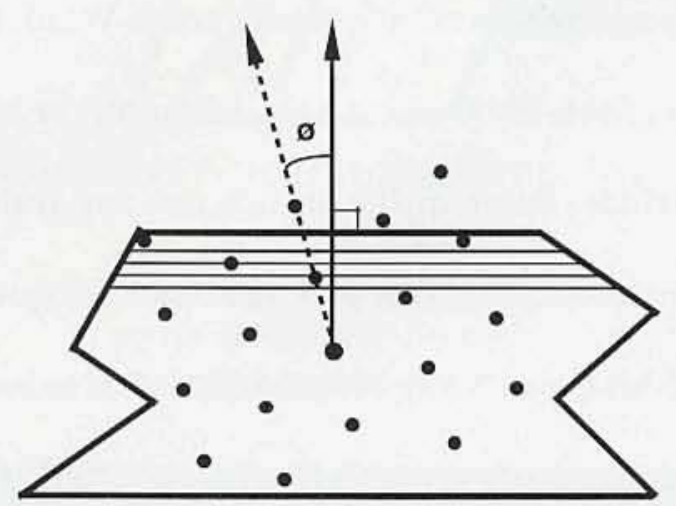

Figure 3: Diagram of a double exposure for the image/diffraction pattern rotation calibration, transferring crystallographic information from the diffraction pattern to the image. 